\title{
Development of time-resolved $x$-ray acoustic method of investigation of crystals
}

Anton Targonskiy ${ }^{1}$, Alexander Blagov $^{2}$, Yan Eliovich ${ }^{1}$, Pavel Prosekov ${ }^{2}$, Yuri Pisarevsky ${ }^{1}$, Andrei Protsenko ${ }^{1}$, Valentin Akkuratov ${ }^{1}$, Michail Kovalchuk ${ }^{2}$

${ }^{1}$ FSRC Crystallography And Photonics RAS, Moscow, Russian Federation, ${ }^{2}$ National Research Center Kurchatov Institute, Moscow, Russian Federation

E-mail: tantva@ya.ru

Development of the method for time-resolved controlling of X-Ray beam parameters by ultrasonic longitudinal vibrations or bending deformations is represented [1]. This method was used to study dynamic of crystal lattice defects structure of the sample in depend on amplitude of mechanical loads (strong vibration) which lead to multiplication of defects and ended by the destruction of the crystals. Intensity distribution analysis in this method is provided by modulation of a lattice parameter or bending of X-ray acoustic element. The distinctive feature of this method is the possibility to carry out precise timeresolved measurements of X-ray rocking curves instead of traditional mechanical system. Experimentally achieved resolutions and angular range of the method are $100 \mathrm{~ms}, 0.1$ arcsec and 100 arcsec respectively [2].

Complex approach based on X-Ray diffraction, electro mechanic and numerical simulation methods was implemented to create high quality $X$-ray acoustic elements (longitudinal or bending) [3].

Evolutions of rocking curves with increasing of amplitude and time of influence of ultrasonic loading for silicon (Si), paratellurite ( $\mathrm{TeO} 2$ ) and lithium fluoride ( $\mathrm{LiF}$ ) crystals were studied. It was found that a form of dependence differ for these crystals. In silicon crystal relative deformation of crystal lattice linearly growing within increasing of amplitude of vibration and after critical point destruction of crystal occur. In lithium fluoride and paratellurite crystals formation of defects that disappear after turned off ultrasound was observed. Temporal and amplitude characteristics of these processes was determined.

One of the main advantages of the proposed method is the possibility of investigating the defect structure of crystals under ultrasonic loading during early stages of nucleation of defects when there is no irreversible deterioration and traditional methods are inapplicable in fact.

This work was performed using the equipment of the Shared Research Center FSRC "Crystallography and Photonics" RAS and was supported by the Russian Ministry of Education and Science, partially supported by the Russian Foundation for Basic Research (proj. № 16-29-14057 ofi_m, 16-32-60045 mol_a_dk).

[1] Blagov A.E., Kohn V.G., Lider V.V., Pisarevskii Y.V. and Kovalchuk M.V. (2005), Journal of experimental and theoretical physics, 101, 5, 770-778 pp

[2] Kovalchuk M.V., Targonskii A.V., Blagov A.E., Zanaveskina I.S. and Pisarevskii Y.V. (2011), Crystallography reports, 56, 5, 828-831pp

[3] Blagov A.E., Darinskii A.N, Prosekov P.A., Targonskii A.V., Pisarevskii Y.V. and Kovalchuk M.V. (2013), Acoustical physics, $59,5,506-512 \mathrm{pp}$

Keywords: diffractometry, $x$-ray acoustooptics, ultrasonic loading 